\title{
Medium access behavior analysis of two-flow topologies in IEEE 802.11 wireless networks
}

\author{
Muhammad Zeeshan* (D) and Anjum Naveed
}

\begin{abstract}
Impact of medium access control (MAC) on throughput of IEEE 802.11-based multi-hop wireless networks is not completely understood despite numerous research efforts. Researchers have explored the MAC interaction of two-flow topologies in order to better understand the MAC behavior of nodes in generic multi-hop wireless network. Prior research has considered two flow interactions under the assumption of same transmission and carrier sensing range. This research extends and completes the existing body of work by relaxing the assumption of same transmission and carrier sensing range to realize more practical and realistic two-flow topologies. Twenty-five unique possible two-flow topologies can exist in general multi-hop wireless networks. The topologies have been classified into six categories based on MAC layer behavior and per flow throughput. Closed-form expressions for occurrence probabilities of the identified categories have been derived with particular observation that carrier sensing range-based categories have high occurrence probability and cannot be ignored. MAC behavior of each category is discussed. It is observed that different transmission and carrier sensing ranges significantly affect the MAC behavior and the throughput of flows. Based on the behavior, exact throughput of the two single hop flows is analytically computed. The results achieved through analysis have been compared with the simulated results to verify the accuracy of analysis. This research will serve as basis for MAC behavior analysis of generic multi-hop wireless networks.
\end{abstract}

Keywords: Wireless mesh networks, Multiple access interference, Two-flow interference analysis

\section{Introduction}

Interference in wireless networks significantly limits the network capacity. Among a set of interfering links using a common frequency channel, transmission of a link is successful only if all other links remain silent for the entire period of transmission. Medium access control (MAC) protocol is employed to arbitrate the access to the wireless channel among competing links. IEEE 802.11 networks use carrier sense multiple access with collision avoidance (CSMA/CA) as MAC protocol. The random access mechanism of CSMA/CA does not ensure interference-free transmissions, specifically when the sender nodes of the interfering links are not within the transmission range of each other. Consequently, many transmission opportunities are wasted when more than one interfering links

\footnotetext{
*Correspondence: muhammad.zeeshan@seecs.edu.pk

National University of Science and Technology (NUST), H-12, Islamabad, Pakistan
}

simultaneously attempt transmissions. Thorough MAC behavior analysis can reveal the impact of interference on the achievable throughput of the interfering links.

Analysis of two-flow topologies is widely used in literature to understand the complex interactions in general multi-hop wireless networks, and analysis of subset of four nodes is suitable to explain all types of interaction that can exist in realistic wireless network deployments. Currently, most deployments of wireless mesh network uses carrier sense multiple access (CSMA) as their MAC protocol. For fully connected topologies where all four nodes or at least both transmitters are within single transmission range, CSMA with or without RTS/CTS demonstrates fair throughput and channel access performance between contending flows. The rest of two-flow topologies in WMN, where both transmitters are not in single transmission range, exhibit severe throughput imbalances between two contending flows and few well-known topologies have

\section{Springer}


been investigated by researchers in the past including hidden and exposed terminal problems. Two-flow topologies in WMN that results in throughput imbalances suffer from problems including severe short- and long-term unfairness between contending flows.

This work further widens and completes the body of work on two-flow interaction analysis for multi-hop wireless network. In this work, two-flow topologies have been classified by separately considering the transmission and the carrier sensing ranges. The interaction between the two single hop flows is considered under CSMA/CA protocol for throughput estimation of two-flow topologies. It is observed that the presence of sender or receiver of interfering link within the carrier sensing range results in a significantly different MAC behavior compared to the presence of the two nodes outside the carrier sensing range. This research divides the two-flow topologies into six categories, depending upon CSMA/CA interaction. Occurrence probability of each category has been computed using spatial analysis. For this purpose, possible geometric area where the nodes of the particular topology can exist has been considered, compared to the overall geometric area of occurrence for two interfering links. Analysis shows that the categories that are based on interference interactions from within the carrier sensing range only have high occurrence probability values (aggregate of 0.69). Finally, throughput achieved by the two links under each category has been computed analytically based on MAC protocol behavior. Analytically computed throughput values have been compared with the simulation throughput values using Opnet-based simulations. The comparison shows near perfect match in analytical and simulated values, suggesting the completeness of the categorization.

The rest of the paper is organized as follows. Section 2 discusses the related work. Section 3 enlists the twoflow categories and possible geometric placement of nodes under each category. Expressions for occurrence probabilities of the categories have been derived in Section 4. Interference type based on MAC protocol and the throughput achieved by each link has been derived for each category in Section 5. Section 6 concludes the paper.

\section{Related work}

Literature relating MAC behavior analysis and capacity estimation can be grouped into three sets. The first set consists of interference models and ensuing MAC behavior analysis that considers interference from within the transmission range. These models consider the impact of interference from different links to be same, irrespective of their relative geometric location [1-4]. The second set comprises of location-based MAC behavior and interference analysis [5-8]. Literature in this group focuses on change in MAC behavior because of changing geometric relation of the interfering links. The final set consists of capacity estimation based on physical characteristics of wireless channel.

Capacity estimation of CSMA-based wireless networks was first performed by Boorstyn et al. [9]. Authors used Markov chain-based model to compute exact throughput in multi-hop CSMA-based wireless networks. However, the analysis was limited to few nodes, given the complexity of computation. Bianchi [10] computed the achievable throughput by individual nodes, given that all interfering nodes are within a transmission range. Bianchi showed that in the absence of hidden node problem [11] and with perfect channel capture, wireless nodes exhibit fairness for all nodes contending for channel access. Although restricted to only one type of two-flow interactions (i.e., coordinated interfering links), Bianchi computed exact throughput values for different IEEE 802.11 DCF mode parameters. Protocol and physical models of interference are well-known interference models [1] that have been used frequently in literature for capacity estimation as well as MAC protocols and channel assignment research. Both models lead to inaccurate interference estimation.

Given a link and its interfering link, the placement of the sender and the receiver of the interfering link within transmission or carrier sensing range defines the MAC behavior and its impact on achievable throughput of the two links. Garetto et al. [5] have analyzed the MAC behavior of the two interfering links under different geometric placements inside and outside transmission range. The authors have categorized the two-flow topologies with different geographic placements into three categories. Under the assumption of the same transmission and carrier sensing range, the analysis carried out by Garetto et al. [5] accurately predicts the impact of MAC behavior and interference on throughput of the two single hop flows. However, the study does not consider the impact of carrier sensing range on the MAC behavior and the resultant throughput. Razak et al. [7] have extended this research by considering separate carrier sensing and transmission ranges; however, the simulation results show that the topologies within the single category do not share the same throughput profile. Furthermore, important categories based on nodes within carrier sensing range have not been considered.

Garetto et al. [5] have considered the two-flow interactions and classified possible topologies into three categories of sender connected (SC), asymmetric incomplete state (AIS), and symmetric incomplete state (SIS) based on MAC behavior and throughput imbalances. In their extended work, Garetto et al. [6] computed per link forwarding capacity for general multi-hop wireless networks using two-flow interactions. In cases where transmission and sensing ranges are considered same, the analytical results accurately predict throughput achieved through 
simulations. However, the model does not capture the impact of interference from links within sensing range. The work has been extended by Razak et al. [7, 8, 12]; however, significant gap exists between analytical and simulated results. Research presented in this document is focused on differentiating between interference introduced from transmission range and from carrier sensing range. This results in new categories that have high occurrence probability in realistic multi-hop wireless networks.

Among capacity estimation literature, Li et al. [13] have performed the throughput analysis of a single access point for IEEE 802.11g radios. Dinitz [14] has proposed distributed algorithms for wireless nodes to achieve optimal throughput in distributed multi-hop wireless networks. The author have used protocol and physical models for interference. Kawade et al. $[15,16]$ have compared the performance of IEEE $802.11 \mathrm{~g}$ and $802.11 \mathrm{~b}$ radios under co-channel interference by considering the physical layer characteristics. The authors have concluded that IEEE 802.11g networks are more resistant to co-channel interference while channel separation improves the performance of both types of networks. Weber et al. [17] have computed the upper and lower bound network capacity for multi-hop wireless networks using different physical channel conditions. The authors have computed maximum physical transmission capacity and optimal number of nodes that achieve the maximum capacity.

$\mathrm{Fu}$ et al. [18] have analyzed the general CSMA proto$\mathrm{col}$ and proposed the concept of cumulative interference model where hidden node problem can be avoided. The authors have also proposed incremental power carrier sensing that can help nodes identify the distance from potential interfering nodes and better plan the transmissions. Vitturi et al. [19] have proposed new techniques for rate adaptation to cater the collision problem and compared the performance with automatic rate fallback technique. The authors have shown that the performance of new techniques is better in terms of retransmissions required. Qiao et al. [20] have also proposed transmit power control and rate adaptation to achieve low energy consumption in IEEE 802.11a/h systems. The objective is to minimize consumed energy, although throughput gains have also been reported.

Focus of this research is the analysis of two-flow interference interactions, impact of geometric location on MAC behavior, and its impact on throughput of the two single hop flows.

\section{Two-flow topology categorization}

Within a multi-hop network, a sender receiver pair (referred as flow throughout the rest of the paper) interacts with multiple flows in the neighborhood. Each interaction impacts the throughput of the flows, resulting in complex chain of interactions. In order to understand such interactions and the resulting impact on the achievable throughput of each flow, it is important to understand the possible interactions between two flows in isolation. Based on this understanding, a general model for wireless interactions in a multi-hop wireless network is conceivable, which can predict the achievable throughput of individual single hop flows. In this section, possible interactions of two flows are categorized based on geometric location of the nodes of the flows. The differences of proposed categorization from the categories defined in prior work $[5,7]$ are highlighted. In the subsequent sections, the achievable throughput is discussed based on the MAC behavior.

\subsection{Terminology}

The Euclidean distance between two nodes $A$ and $B$ is given as $d(A, B)$. A node can have three possible placements with reference to another node depending upon the signal strength received from the other node. If node $B$ is placed around node $A$ such that it can successfully decode the transmissions from node $A$, then the node $B$ is within the transmission range (TR) of node $A$, i.e., $d(A, B) \leq$ TR. Such placement is referred as connected in this paper. On the other hand, if node $B$ can sense the channel to be busy when node $A$ transmits but cannot successfully decode the information because of weak radio signals, then the node $B$ is outside transmission range but within the carrier sensing range (CSR) of node $A$, i.e., $\mathrm{TR}<d(A, B) \leq \mathrm{CSR}$. This placement is referred as sensing. Finally, if node $B$ cannot sense the transmissions of node $A$, then node $B$ is outside the carrier sensing range of node $A$, i.e., $d(A, B)>$ CSR. This placement is referred as disconnected. The placement is referred as not connected if it is either sensing or disconnected. The placements are referred as interference interactions throughout the rest of the paper.

Consider two single hop flows $A a$ and $B b$ where $A$ and $B$ are the transmitting nodes while $a$ and $b$ are the respective receiving nodes. The receiving nodes are within the transmission range of the respective transmitting nodes. Four interference interactions $A B, a b, A b$, and $B a$ exist between the nodes of the two flows, as shown in Fig. 1. Interference interaction $A B$ is between the two transmitters and is referred as sender interaction. On the similar lines, the interaction $a b$ is referred as receiver interaction while the interactions $a B$ and $A b$ are referred as sender receiver interactions. A two-flow topology is considered symmetric if the interference interactions $A b$ and $B a$ are of same type, i.e., if $A b$ is sensing then $B a$ is also sensing.

\subsection{Two-flow topologies}

Based on the interference interactions, there are a total of $3^{4}(81)$ possible two-flow topologies while 53 of these topologies are unique. Given the restriction that the 


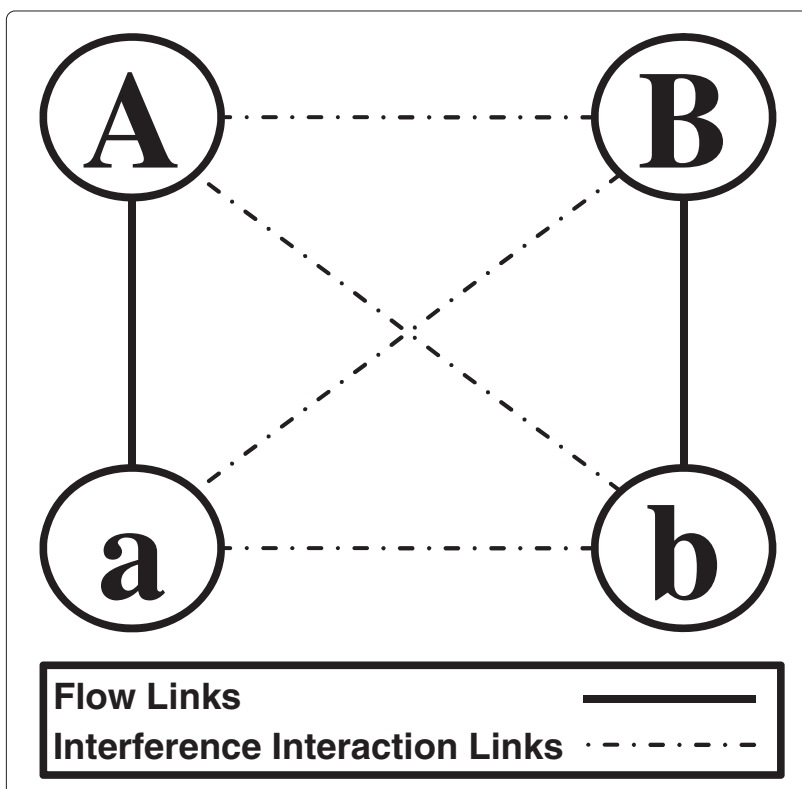

Fig. 1 Two-flow interaction topology. Four interference interactions $A B, a b, A b$, and $B a$ exist between the nodes of the two flows transmitters of flows must be within the transmission range of the respective receivers and the fact that carrier sensing range is $\approx 2.7$ times the transmission range (through simulations and experimentations), only 25 topologies are physically realizable in a multi-hop wireless network. Remaining 28 topologies have zero occurrence probability. The 25 unique possible topologies have been classified into six categories, depending upon the types of the four interference interactions and the MAC behavior. The following sections explain the interference interactions of the categories.

\subsubsection{Sender connected (SC)}

Sender connected category represents the topologies where the transmitters $A$ and $B$ of the single hop flows $A a$ and $B b$ are within the transmission range of each other. That is:

$$
d(A, B) \leq \mathrm{TR}
$$

The remaining three interference interactions are not significant in this case. Seven topologies belong to this category and are shown in Fig. 2. Node placement of a sample topology is shown in Fig. 3. This category exists in the classification by Garetto et al. [5] with the same name. Razak et al. [7] have divided this category into two

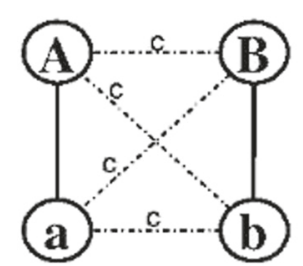

a

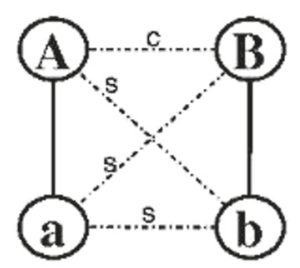

d

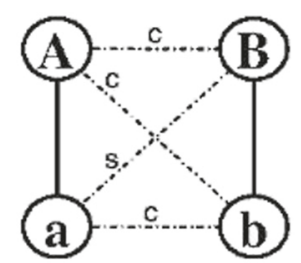

b

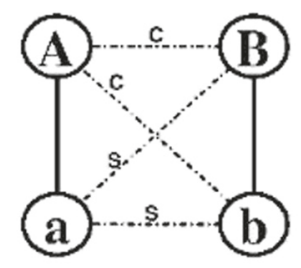

e

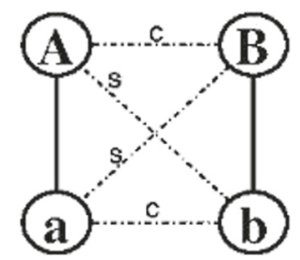

C

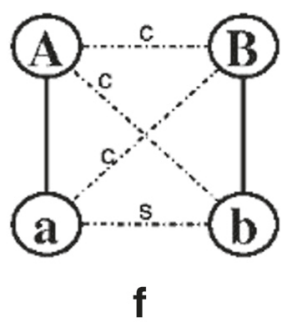

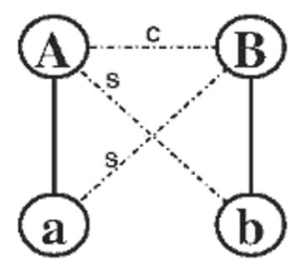

Fig. 2 Sender connected topologies 


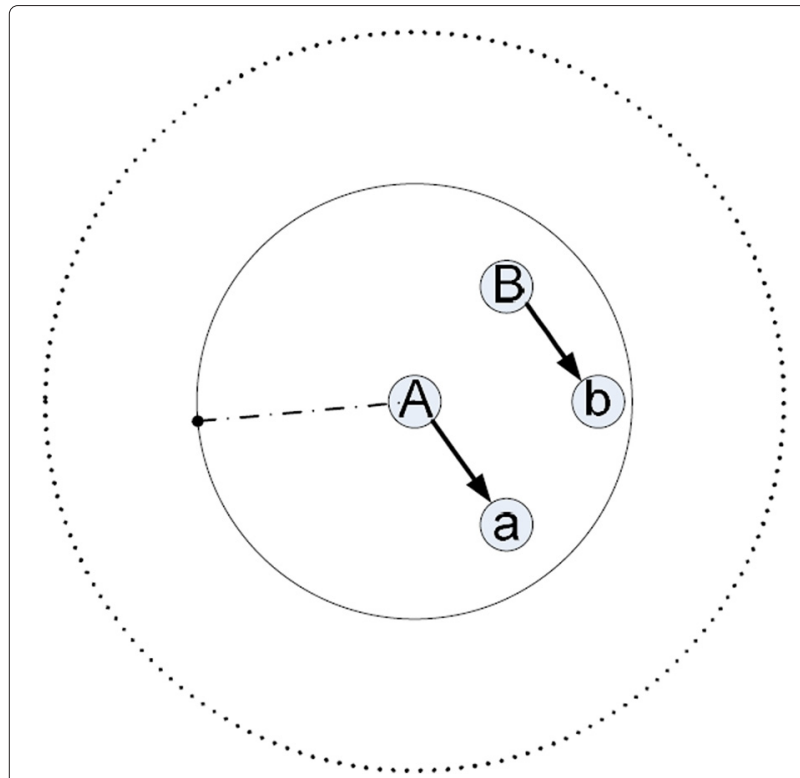

Fig. 3 Sample sender connected node placement

categories of sender connected symmetric interference, and sender connected asymmetric interference. However, as shown in the subsequent section, the MAC behavior and resulting throughput profile of all topologies belonging to this category are the same and form a single category.

\subsubsection{Symmetric sender receiver connected (SSRC)}

If the interference interaction $A B$ is not connected and interference interactions $A b$ and $a B$ are both connected, then the topology belongs to the category of symmetric sender receiver connected. The topologies fulfill following criteria.

$$
\begin{aligned}
& d(A, B)>\mathrm{TR} \\
& d(a, B) \leq \mathrm{TR} \quad \& \quad d(A, b) \leq \mathrm{TR}
\end{aligned}
$$

Two topologies belong to this category as shown in Fig. 4. Sample node placement is shown in Fig. 5. Garetto et al. in their extended work [6] refer to this category as near hidden terminals. The same category exists with the name

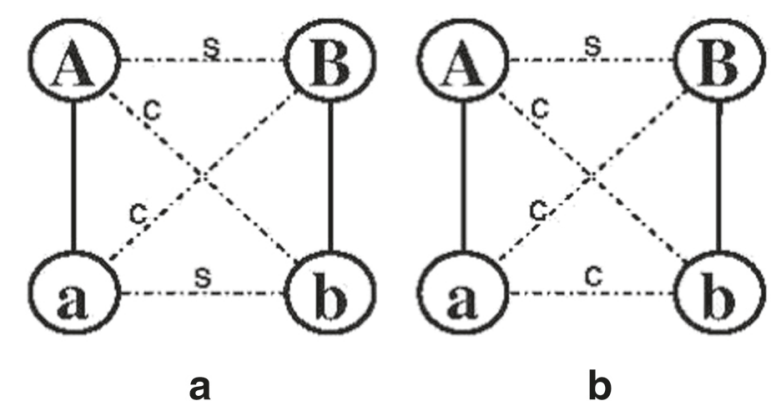

Fig. 4 Symmetric sender receiver connected topologies

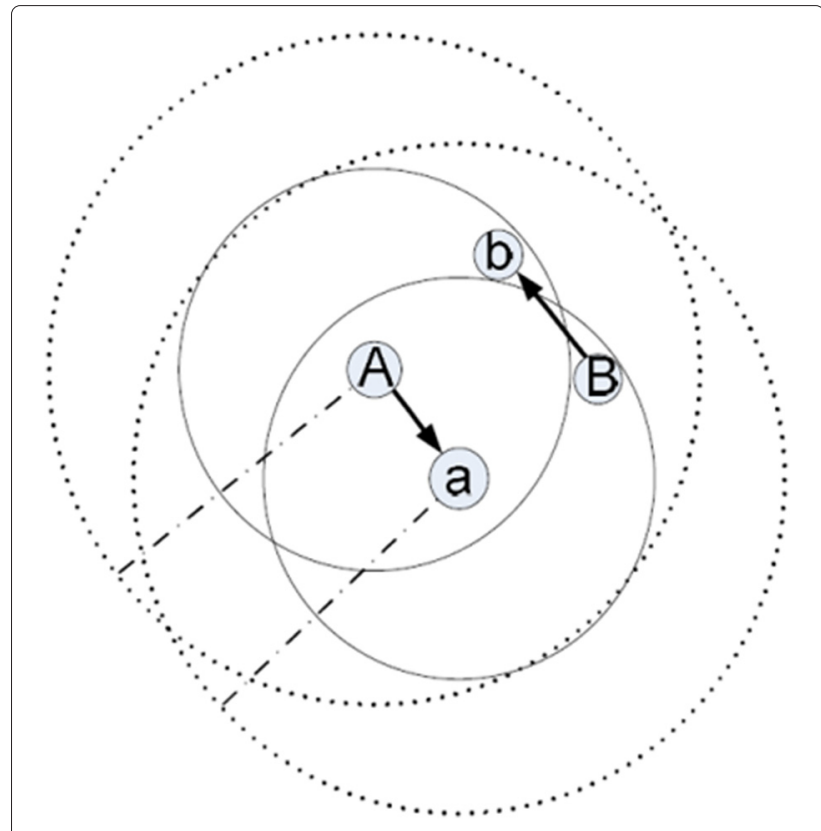

Fig. 5 Sample SSRC node placement

of symmetric incomplete state in their original work [5] as well as in the work by Razak et al. [7] but contains an additional topology. The additional topology is not realistic when $\mathrm{CSR} \geq 2 * \mathrm{TR}$.

\subsubsection{Asymmetric sender receiver connected (ASRC)}

If the interference interaction $A B$ is not connected and one of the interference interactions $A b$ and $a B$ is connected while other interaction is either disconnected or sensing, then the resulting topology belongs to the category of asymmetric sender receiver connected. Three topologies belong to this category as shown in Fig. 6 and a sample node placement of ASRC is shown in Fig. 7. The topologies belonging to this category fulfill the distance criteria of

$$
\begin{aligned}
& d(A, B)>\mathrm{TR} \\
& d(a, B) \leq \mathrm{TR} \quad \& \quad d(A, b)>\mathrm{TR}
\end{aligned}
$$

This category exists with the name of asymmetric incomplete state in the categorization of Garetto et al. [5] and Razak et al. [7]. However, an additional topology (Fig. 6a) is part of this category in the proposed categorization because of the different sensing range and transmission range.

\subsubsection{Receiver connected ( $R C)$}

This category consists of the topologies where the interference interactions $A B, a B$, and $A b$ are not connected (i.e., either sensing or disconnected) and the interference 


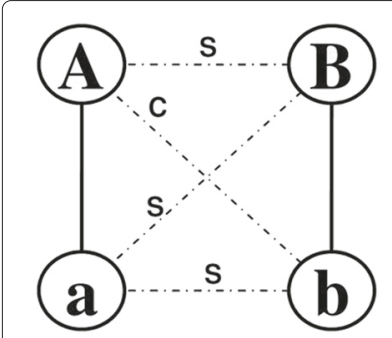

a

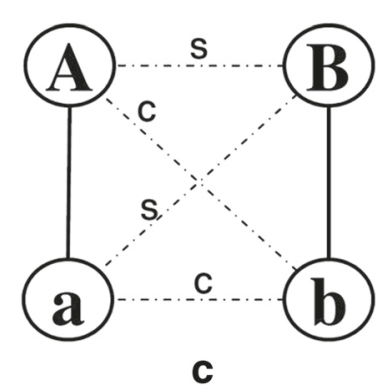

Fig. 6 Scenarios of asymmetric sender receiver connected topologies

interaction $a b$ is connected. The Euclidean distances of interference interactions are

$$
\begin{aligned}
& d(A, B)>\mathrm{TR} \\
& d(a, B)>\mathrm{TR} \quad \& \quad d(A, b)>\mathrm{TR} \\
& d(a, b) \leq \mathrm{TR}
\end{aligned}
$$

Two topologies belong to this category as shown in Fig. 8a, b. A sample node placement is shown in Fig. 9. Razak et al. [7] have referred to this category as interfering

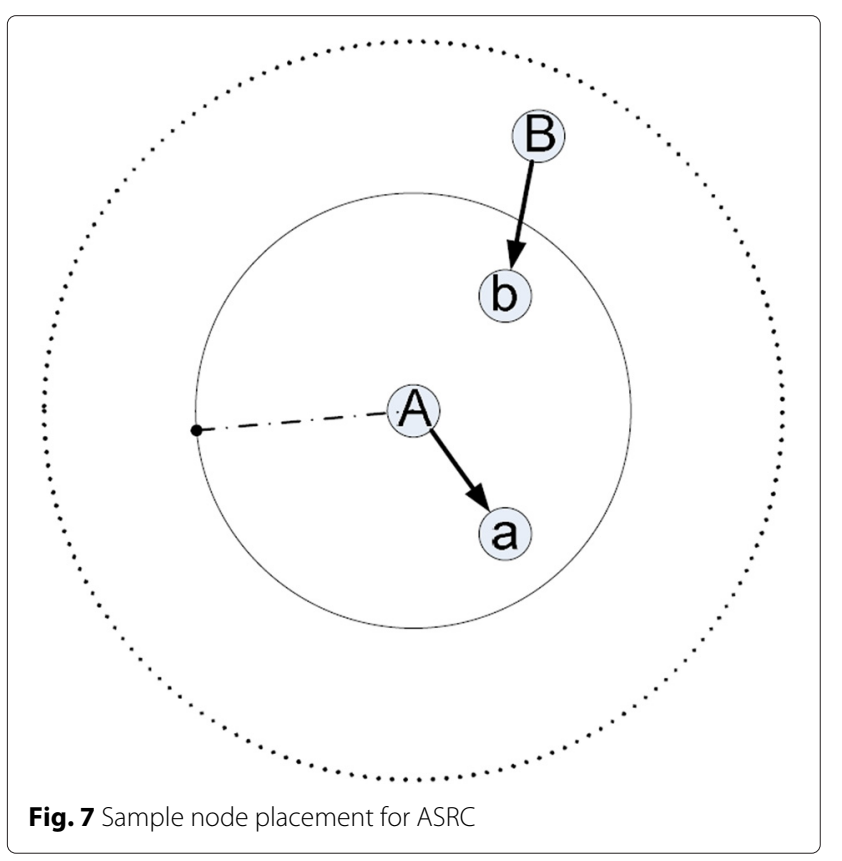

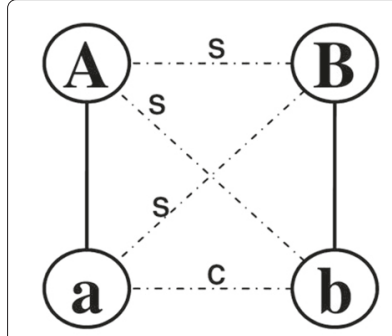

a

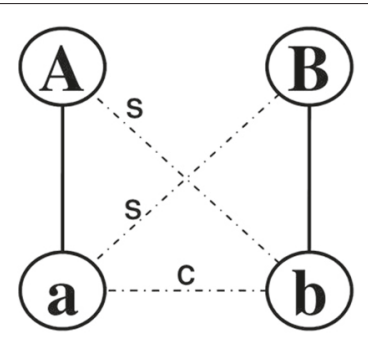

b
Fig. 8 Scenarios of receiver connected topologies

destination incomplete state. However, a different MAC behavior has been observed in the proposed work compared to the one reported by Razak et al. This is explained in the subsequent section.

\subsubsection{Symmetric not connected (SNC)}

This is a new category and does not exist in any of the prior categorization. If none of the four interference interactions is connected and the sender receiver interactions $a B$ and $A b$ are symmetric, then the topologies belong to the category of symmetric not connected. Seven topologies belong to this category and are shown in Figs. 10 and 11. Sample node placement is also shown in Fig. 12. The topologies satisfy the distance criteria of

$$
\begin{aligned}
& d(A, B)>\mathrm{TR} \\
& (\mathrm{TR}<d(a, B) \leq \mathrm{CSR} \& \mathrm{TR}<d(A, b) \leq \mathrm{CSR}) \quad \mathrm{OR} \\
& (d(a, B)>\mathrm{CSR} \quad \& \quad d(A, b)>\mathrm{CSR}) \\
& d(a, b)>\mathrm{TR}
\end{aligned}
$$

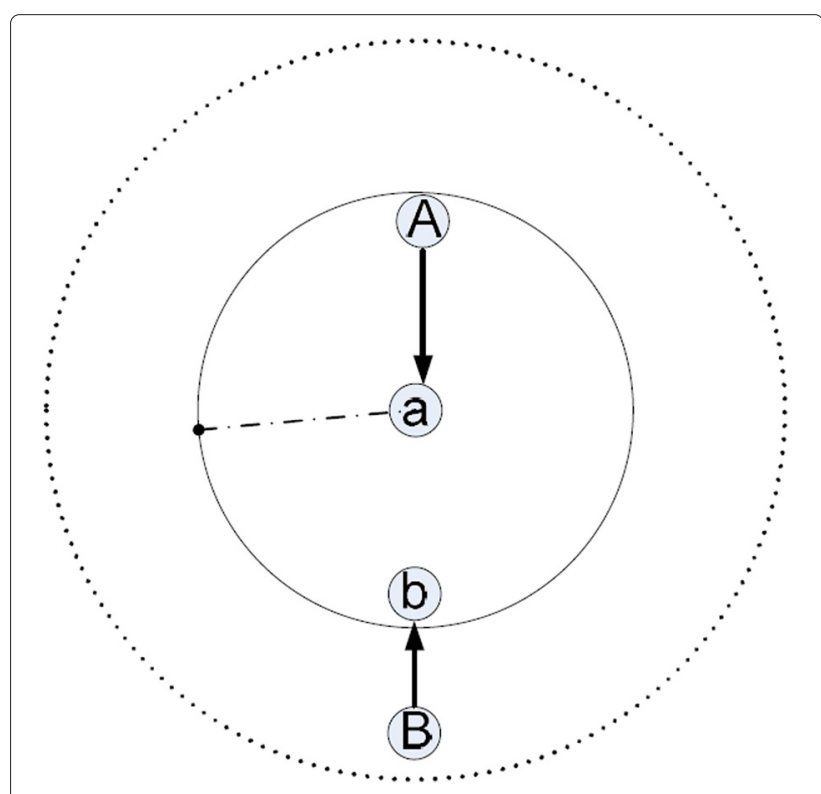

Fig. 9 Sample node placement for RC 


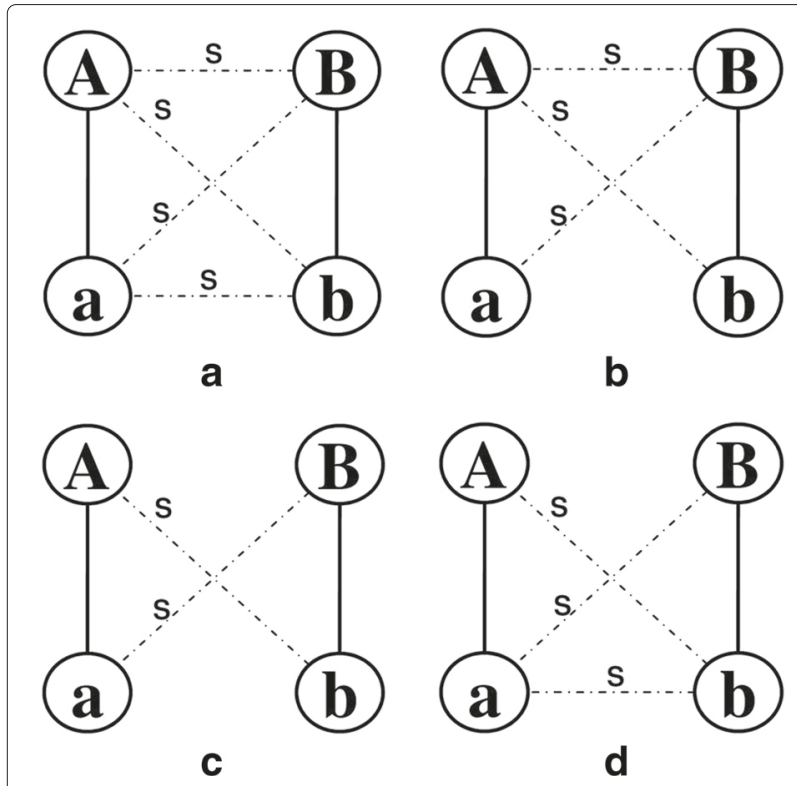

Fig. 10 Scenarios of symmetric not connected topologies

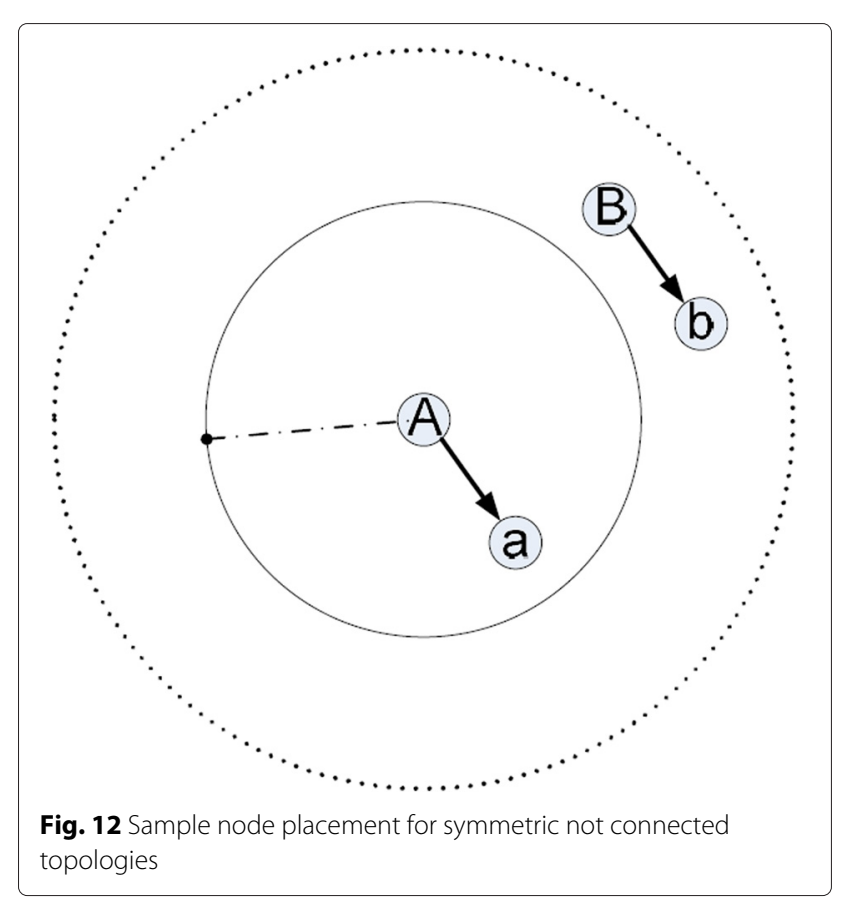

is a geometric representation of a sample topology. The topologies satisfy the interference interaction distance criteria of

$$
\begin{aligned}
& d(A, B)>\mathrm{TR} \\
& \mathrm{TR}<(d(a, B) \leq \mathrm{CSR} \quad \& \quad d(A, b)>\mathrm{CSR} \\
& d(a, b)>\mathrm{TR}
\end{aligned}
$$

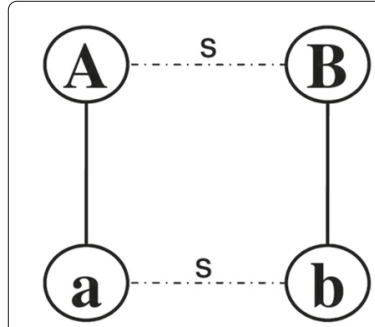

a

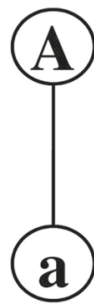

a.

\section{b}
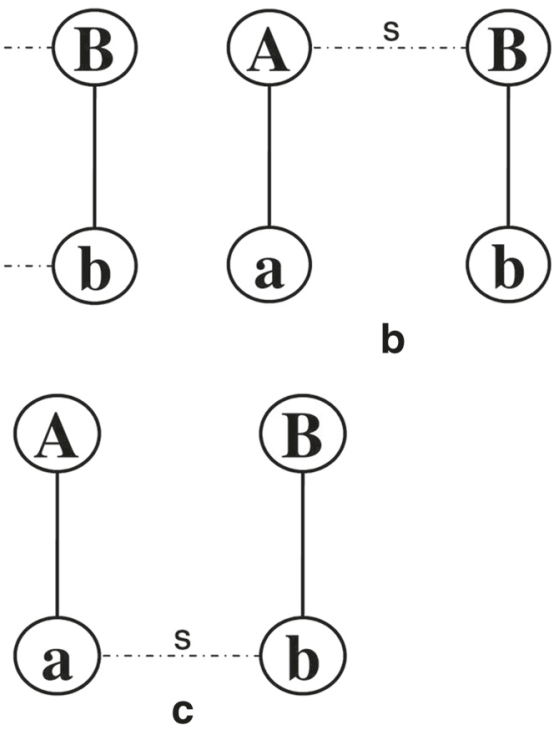

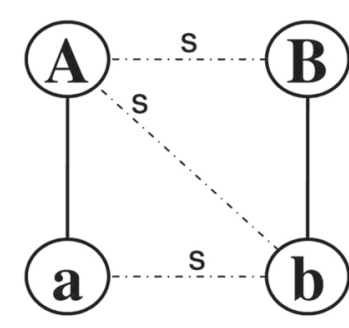

a

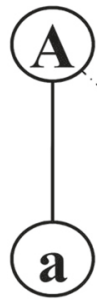

a

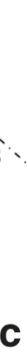

C

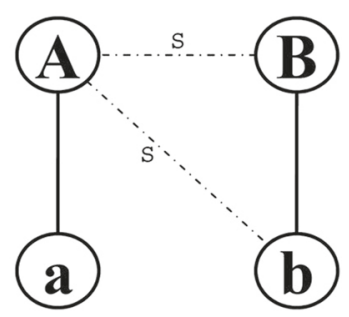

b

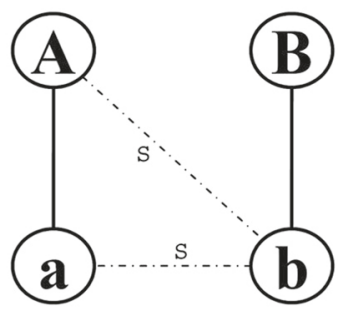

d

Fig. 13 Scenarios of asymmetric not connected topologies

Fig. 11 Scenarios of symmetric not connected topologies 


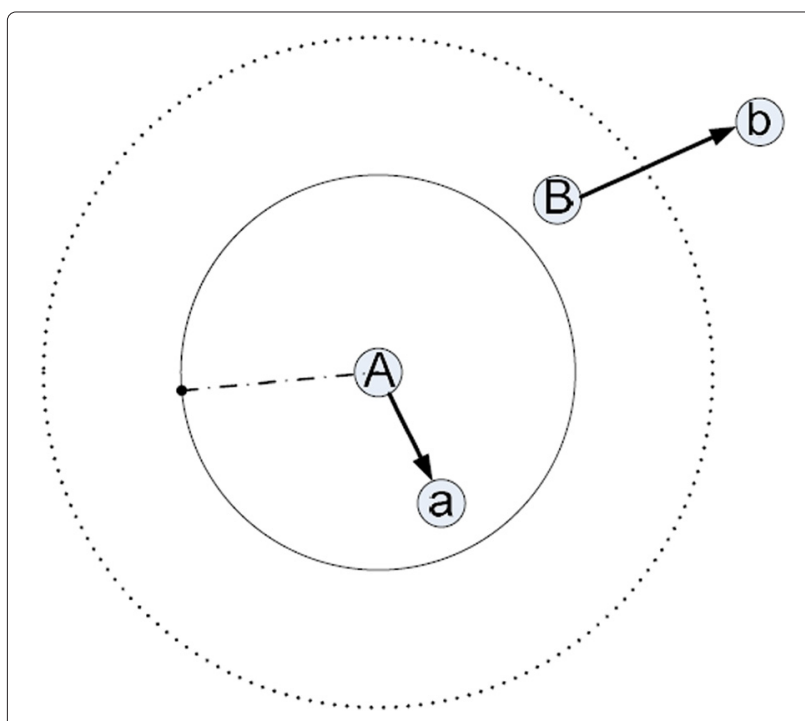

Fig. 14 Sample node placement for asymmetric not connected topologies

\section{Category occurrence probabilities}

How frequently the topologies belonging to each category can exist in a general multi-hop wireless network? Specifically, what is the occurrence probability of the newly identified categories? Answers to these questions are important in identifying the impact of each category on interference profile of the links in general multi-hop wireless networks. Geometric analysis has been employed to find out the occurrence probability of the categories. Perfect circular disks are assumed for area under transmission range, carrier sensing range, and the network with the disk radii defined as $r_{\mathrm{tr}}, r_{\mathrm{csr}}$, and $r_{\mathrm{n}}$, respectively. Network radius is assumed to be $r_{\mathrm{n}}=0.5 \times\left(2 \times r_{\mathrm{tr}}+\right.$ $\left.r_{\mathrm{csr}}\right)$ which covers maximum possible distance for a valid placement of the nodes such that the resulting flows are interfering. Note that at times, the carrier sensing range of nodes can be outside the total network area; however, the ratio of area of interest and the total area remains unaffected.

For each category, four interference interactions $A B$, $A b, a B$, and $a b$ are considered individually. For each pair of nodes within the interference interaction, one node is assumed to be at a fixed location. The area around the first node where the second node can possibly exist is computed, given the placement constraints introduced by the specific category. Under the assumption of circular disk ranges, the area is mostly equivalent to either the area of a disk or the area of intersection of two disks with known radii. The ratio of computed area to the maximum possible network area gives the probability of occurrence of the interference interaction.
Multiplying the occurrence probabilities of four individual interactions gives the occurrence probability of the category.

The expressions for the disk area and the area of interaction of two disks are frequently used throughout the computations. The expression for area of circular disk with radius $r$ using onion method is given as

$$
\int_{0}^{r} 2 \pi x d x
$$

The expression for area of intersecting circles of same radius $r$ and distance between radii as $d$ is given as

$$
2 d^{2} \cos ^{-1}\left(\frac{d}{2 r}\right)-\frac{d}{2} \sqrt{4 r^{2}-d^{2}}
$$

The occurrence probability of each category is computed in the subsequent section using the two listed expressions. For sake of brevity, the final expression for probability of each category is given with brief description of the expression.

\subsection{Sender connected}

The transmitters of both flows must be within the transmission range of each other. This leads to a disk area with radius $r_{\mathrm{tr}}$. The probability of $A B$ to be connected is achieved by integrating $\frac{2 x}{r_{\mathrm{n}}^{2}}$ over the interval 0 to $r_{\text {tr }}$. Given that $A B$ is connected, maximum distance between nodes $A$ and $b(a$ and $B)$ is $2 r_{\mathrm{tr}}$. The probability of two events can be achieved by integrating $\frac{2 x}{r_{\mathrm{n}}^{2}}$ over the interval 0 to $2 r_{\mathrm{tr}}$. Finally, the two receivers can be located anywhere in the network. The occurrence probability of sender connected category is given as

$$
P_{\mathrm{SC}}=\int_{0}^{r_{\mathrm{csr}}} \int_{0}^{2 r_{\mathrm{tr}}} \int_{0}^{2 r_{\mathrm{tr}}} \int_{0}^{r_{\mathrm{tr}}} \frac{2 w}{r_{\mathrm{n}}^{2}} \frac{2 x}{r_{\mathrm{n}}^{2}} \frac{2 y}{r_{\mathrm{n}}^{2}} \frac{2 z}{r_{\mathrm{n}}^{2}} d w d x d y d z
$$

\subsection{Symmetric sender receiver connected}

Topologies belonging to SSRC category have the interference interaction $A B$ as sensing, i.e., $r_{\mathrm{tr}}<d(A, B) \leq$ $r_{\mathrm{csr}}$. The interactions $A b$ and $a B$ should be connected. Therefore, the distance $d(A, B)$ is restricted to $2 * r_{\mathrm{tr}}$. Furthermore, the receiver $a$ (or receiver $b$ ) should be within the transmission range of sender $A$ (or sender $B$ ) as well as $B(A)$. Consequently, the possible placement area of $a$ around node $B$ is given by the area of intersection of the two circles with radius $r_{\text {tr }}$ and centers separated by the distance $r_{\mathrm{tr}}$. The area is given by expression 2 where 
$d(A, B)>r_{\text {tr }}$ results in maximum distance between $a$ and $b$ to be $\sqrt{3} r_{\text {tr }}$. The probability of the category is given as

$$
\begin{aligned}
P_{\mathrm{SSRC}}= & \left(2 d^{2} \cos ^{-1}\left(\frac{d}{2 r}\right)-\frac{d}{2} \sqrt{4 r^{2}-d^{2}}\right)^{2} \\
& \times \int_{0}^{\sqrt{3} r_{\mathrm{tr}}} \int_{r_{\mathrm{tr}}}^{2 r_{\mathrm{tr}}} \frac{2 w}{r_{\mathrm{n}}^{2}} \frac{2 z}{r_{\mathrm{n}}^{2}} d w d z
\end{aligned}
$$

where $r=d=r_{\text {tr. }}$.

\subsection{Asymmetric sender receiver connected}

In this category the condition $r_{\mathrm{tr}}<d(A, B) \leq r_{\mathrm{csr}}$ holds. Similarly, $d(A, b) \leq r_{\text {tr }}$ and area around $A$ where $b$ can exist is given by expression 2 . However, for interaction $a B$, $r_{\mathrm{tr}}<d(a, B)<r_{\mathrm{n}}$. Maximum possible distance between $a$ and $b$ can be $2 r_{\text {tr }}$. The probability expression is given as

$$
\begin{aligned}
P_{\mathrm{ASRC}}= & \left(2 d^{2} \cos ^{-1}\left(\frac{d}{2 r}\right)-\frac{d}{2} \sqrt{4 r^{2}-d^{2}}\right) \\
& \times \int_{0}^{2 r_{\mathrm{tr}}} \int_{r_{\mathrm{tr}}}^{r_{\mathrm{n}}} \int_{r_{\mathrm{tr}}}^{2 r_{\mathrm{tr}}} \frac{2 w}{r_{\mathrm{n}}^{2}} \frac{2 y}{r_{\mathrm{n}}^{2}} \frac{2 z}{r_{\mathrm{n}}^{2}} d w d y d z
\end{aligned}
$$

where $r=d=r_{\text {tr }}$.

\subsection{Receiver connected}

The two receivers should be in transmission range of each other. The interactions $A b$ and $a B$ are sensing. Given the fact that $d(a, b)<r_{\mathrm{tr}}$, the conditions $r_{\mathrm{tr}}<d(a, B)<2 r_{\mathrm{tr}}$ and $r_{\text {tr }}<d(A, b)<2 r_{\text {tr }}$ must hold. The probability of the category is given as

$$
\begin{aligned}
P_{\mathrm{RC}}= & \left(2 d^{2} \cos ^{-1}\left(\frac{d}{2 r}\right)-\frac{d}{2} \sqrt{4 r^{2}-d^{2}}\right) \\
& \times \int_{r_{\mathrm{tr}}}^{2 r_{\mathrm{tr}}} \int_{r_{\mathrm{tr}}}^{2 r_{\mathrm{tr}}} \int_{r_{\mathrm{tr}}}^{r_{\mathrm{n}}} \frac{2 w}{r_{\mathrm{n}}^{2}} \frac{2 y}{r_{\mathrm{n}}^{2}} \frac{2 z}{r_{\mathrm{n}}^{2}} d w d y d z
\end{aligned}
$$

where $r=d=r_{\text {tr }}$.

\subsection{Symmetric not connected}

The four interference interactions in this category are sensing with only restriction on maximum possible area. The probability is given as

$$
P_{\mathrm{SNC}}=\left(\int_{r_{\mathrm{tr}}}^{r_{\mathrm{n}}} \frac{2 z}{r_{\mathrm{n}}^{2}} d z\right)^{4}
$$

\subsection{Asymmetric not connected}

In this case, the interference interaction $a B$ must be disconnected. The area of interest for this case is approximated by integrating $2 z$ over the range $r_{\mathrm{n}}$ to $r_{\mathrm{csr}}$, which is approximately equal to the area outside CSR. The occurrence probability of the category is given as

$$
P_{\mathrm{ANC}}=\int_{r_{\mathrm{n}}}^{r_{\mathrm{csr}}} \int_{r_{\mathrm{tr}}}^{r_{\mathrm{csr}}} \int_{r_{\mathrm{tr}}}^{r_{\mathrm{n}}} \int_{r_{\mathrm{tr}}}^{r_{\mathrm{n}}} \frac{2 w}{r_{\mathrm{n}}^{2}} \frac{2 x}{r_{\mathrm{n}}^{2}} \frac{2 y}{r_{\mathrm{n}}^{2}} \frac{2 z}{r_{\mathrm{n}}^{2}} d w d x d y d z
$$

\subsection{Occurrence probability values}

The probability equations are dependent only on the transmission and carrier sensing ranges. All probabilities are closed-form expressions and can easily be computed. To verify the correctness of expressions, a program has been implemented in Java. The program considers a fixed network area with points arranged in the area as uniform grid. Four nodes are placed on all possible points, and their interference interactions are computed to identify the category of the topology. Non-possible topologies have been eliminated and the remaining $\mathrm{n}$ normalized to attain the occurrence probabilities of the categories. The probability values achieved through the program and the computed values using probability expressions have been plotted in Fig. 15. The plot shows excellent match between all computed values and the values achieved through the program.

Figure 15 shows that the occurrence probability is significantly high for the categories that are purely based on interactions because of carrier sensing range ( $\mathrm{SNC}=0.45$ and $\mathrm{ANC}=0.24)$. In the subsequent section, we show that these interference interactions significantly affect the throughput of interfering links. Therefore, the categories cannot be ignored.

\section{Interference and throughput analysis}

IEEE 802.11 wireless interfaces use carrier sense multiple access (CSMA) with collision avoidance (CA) protocol for acquiring wireless channel access. This section starts with brief explanation of the CSMA/CA protocol as used in IEEE 802.11 (Only extended mode is explained). Parameters affecting the throughput are discussed, and the throughput expressions derived by Bianchi [10] and Kumar et al. [21] are listed. Subsequently, the expressions for the parameters in the throughput expressions are

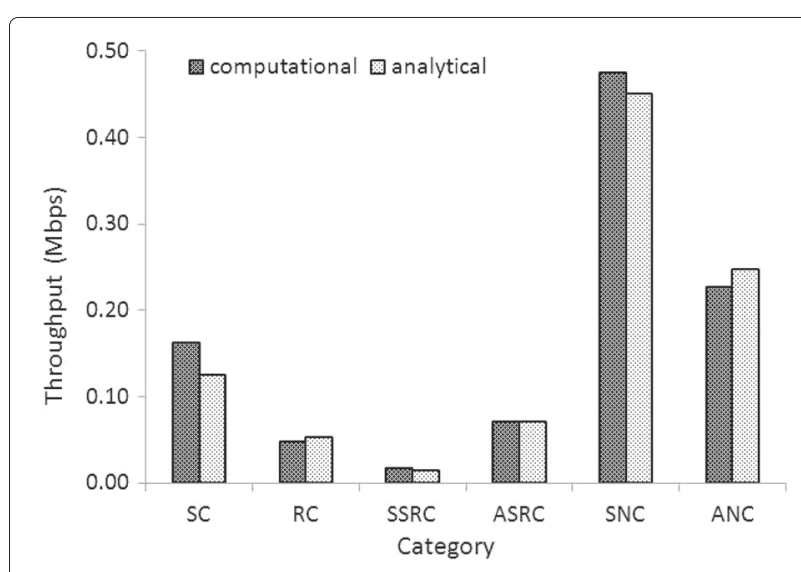

Fig. 15 Occurrence probabilities of categories 
derived for the two flows for each category, throughput for different packet sizes is computed, and the computed values are compared with simulated results to highlight the accuracy of the categorization and the throughput analysis.

\subsection{CSMA/CA protocol behavior}

In IEEE 802.11 MAC, time is considered to be slotted and the slot interval is represented by $\sigma$. Based on the CSMA protocol, when a node has data to transmit, it sets a back-off counter by selecting a random value from the range $\left[0, W_{i}-1\right]$. For the first attempt, $W_{0}=16$ for IEEE $802.11 \mathrm{a} / \mathrm{g}$ radios. The counter is decremented whenever the channel is found idle for the slot interval. If the channel is not idle because of an ongoing transmission from a neighboring node, the back-off counter freezes. When the counter reaches zero and the channel is idle, the node initiates transmission by sending readyto-send (RTS) frame. The transmitting node waits for the response from intended receiver, which is in the form of clear-to-send (CTS) frame. If the CTS is not received within a certain period of time (SIFS +2 *propagation delay), the RTS is assumed to be lost (due to collision or because of busy channel at receiver end). In case of collision, the node resets the back-off counter by selecting a random value from the range $\left[0,2^{i} * W_{0}-1\right]$ where $i$ is the number of retransmission attempt and is known as a back-off stage. The entire procedure of channel access is repeated. If the CTS is received, the channel is reserved for the particular transmission and the node proceeds with transmission of data packet, followed by ACK from a receiver. The four frames RTS, CTS, DATA, and ACK are separated by Short Inter-Frame Space (SIFS) while ACK frame is followed by DCF Inter-Frame Space (DIFS). In case of IEEE 802.11g radios, every frame is followed by signal extension, which is idle interval of $6 \mu \mathrm{s}$, necessary for proper reception of signal. The nodes other than the transmitter and receiver that correctly receive the RTS or CTS frame set the NAV for remaining period of transmission and freeze their activity on the channel.

Assuming that the transmitting nodes are continuously back logged, a wireless node can find the channel in one of the following four states: (i) idle with no transmission going on, (ii) busy because of transmission of another node, (iii) successful transmit of the node itself, (iv) unsuccessful transmit of the node itself with transmitted frame colliding with transmission from another frame. Throughout the analysis, it is assumed that if a packet is received collision free, it can successfully be decoded and no errors occur because of channel noise. Using random back-off mechanism of the CSMA protocol, the probability $\tau$ that a node transmits following an idle slot is given by the equation [21].

$$
\tau=\frac{2(1-2 p)\left(1-p^{m+1}\right)}{q\left(1-p^{m+1}\right)+W_{0}\left(1-p-p(2 p)^{m^{\prime}}\left(1+p^{m-m^{\prime}} q\right)\right)}
$$

where $q=1-2 p, m$ is the maximum back-off stage, and $m^{\prime}$ is the stage when upper limit of the range for random back-off reaches its maximum value. $p$ is the conditional packet loss probability due to collision. Keeping in view the abovementioned states, the throughput (in pkts/s) of a node is given as [5]

$$
T=\frac{\tau(1-p)}{\tau(1-p) T_{s}+\tau p T_{c}+(1-\tau)(1-b) \sigma+(1-\tau) b T_{b}}
$$

where $b$ is the probability that a node finds a slot to be busy while $T_{s}, T_{c}$, and $T_{b}$ are the average durations of successful transmission, collision, and busy interval, respectively, as observed by the node. For all two-flow categories, the values of $T_{s}, T_{c}$, and $T_{b}$ are known. Throughput of a sender can be computed, given the values of busy probability $b$ and conditional packet loss probability $p$. Both values are dependent upon the interference interactions of the categories and need to be computed for individual categories. The values of all known parameters for extended access mode of IEEE $802.11 \mathrm{~g}$ radio assuming homogeneous network and extended rate physical layer are given in Table 1. For other radio types, the values can be updated to get the throughput results.

Table 1 Parameters (ERP IEEE 802.11g)

\begin{tabular}{ll}
\hline Parameter & Value \\
\hline Data rate & 54 Mbps, 216 bits/symbol \\
Basic rate & 6 Mbps, 24 bits/symbol \\
$W_{0}$ & 16 \\
$W_{\max }$ & 1024 \\
$m$ & 6 \\
$m^{\prime}$ & 6 \\
Symbol duration & $4 \mu \mathrm{s}$ \\
$\sigma$ & $9 \mu \mathrm{s}$ \\
SIFS & $10 \mu \mathrm{s}$ \\
DIFS & $28 \mu \mathrm{s}$ \\
PHY & $20+6 \mu$ s (including signal extension) \\
RTS, CTS, MAC, ACK & $20,14,34,14$ bytes at basic rate \\
& $($ ceil(bits/(bits/sym)) $\times 4 \mu s)+$ PHY \\
DATA & at data rate, measured in symbol duration \\
$T_{S}$ & RTS + CTS + DATA + ACK + \\
& 3 SIFS + DIFS \\
$T_{C}$ & RTS + DIFS \\
\hline
\end{tabular}


In the following, the MAC behavior based on interference interactions for the identified categories is explained and the known parameters are computed to compute the achievable throughput for both flows of each category.

\subsection{Sender connected}

MAC behavior of this category is simplest to understand. The senders $A$ and $B$ of the two flows are within the transmission range of each other; therefore, the RTS packet transmitted by sender $A$ is successfully received by sender $B$ and vice versa. Therefore, sender $B$ sets its network allocation vector (NAV) and freezes its activity until the transmission by sender $A$ is complete. The occurrence of busy event for any flow is equal to the occurrence of the transmission event of the alternate sender, which has the probability $\tau$. Therefore, busy probability of any sender is given by $b=\tau$. Collision of RTS for any sender occurs only when the two senders simultaneously start RTS transmission following random back-off. Therefore, conditional packet loss probability of any flow is given by $p=\tau$. Channel busy time $T_{b}$ is equal to $T_{s}$. Replacing these values in Eqs. 9 and 10 gives the value of throughput of a single flow. Based on random access of CSMA/CA and completely symmetric channel view, the throughput of both flows is equal. Bianchi [10] computed the throughput for flows under this category and the results of two computations are same. Figure 16 shows the throughput achieved by the two flows in sender connected category for different packet sizes. It can be seen that the analytically computed throughput perfectly matches the simulated throughput for all packet sizes. Maximum achievable throughput for any flow is $10.68 \mathrm{Mbps}$ for the packet size of 1500 bytes.

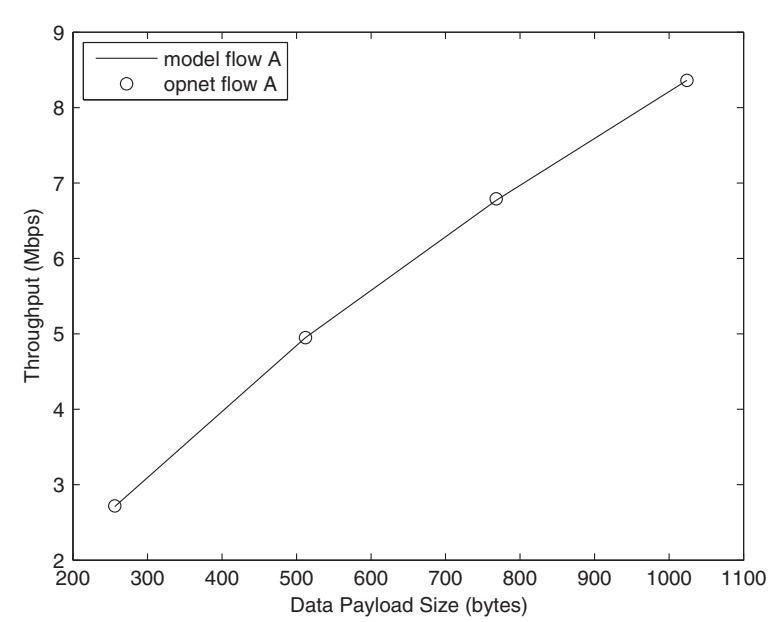

Fig. 16 Throughput (sender connected flows)

\subsection{Symmetric sender receiver connected (SSRC)}

The senders $A$ and $B$ of the two flows in this category (and all subsequent categories) are not within the transmission range of each other. Therefore, the RTS frame transmitted by sender $A$ is not successfully decoded by sender $B$ and vice versa. However, the channel is sensed busy during RTS transmission, preventing the other sender from initiating a transmission. This is different from SIS category proposed by Garetto et al. [5] where senders are assumed to be outside the sensing range and cannot sense the RTS transmitted by sender of the alternate flow. The receivers of both flows are within the transmission range of the alternate senders, i.e., interference interactions $A b$ and $a B$ are connected. This means that the receivers can successfully decode the RTS frame transmitted by the alternate senders resulting in setting NAV at alternate receiver. Similarly, senders can successfully decode the CTS packet transmitted by the alternate receivers. Therefore, collision can only occur if one sender starts transmission of RTS during the idle interval between RTS and CTS transmission of the alternate flow.

To compute the throughput of the flows in SSRC category, we start with busy probability. Busy probability $b$ of each flow is equal to the successful transmission probability of the alternate flow. To compute successful transmission probability and the conditional packet loss probability, we adopt the method used by Garetto et al. [5] for analysis of SIS category (see section 5 of [5]). Collision occurs if sender $B$ starts RTS transmission during signal extension + SIFS $(6 \mu \mathrm{s})$ interval between RTS and CTS frames of flow $A a$. This depends upon two factors: (i) number of transmission opportunities where collision can occur, which is given by $f=\operatorname{ceil}((\operatorname{SIFS}+6) / \sigma)$ as shown in Fig. 17 and (ii) the back-off stage of sender $B$, which defines the probability of transmission in a given slot for sender $B$. The probability of transmission is given by $\gamma_{i}=\frac{2}{1+W_{i}}$ where $i$ is the back-off stage. The interaction of the two senders during interval $f$ can be modeled as two-dimensional Markov model. Each state of the model represents the back-off stage of both senders, resulting in $m^{2}$ states. In a general state $(i, j)$, the transition probabilities are given by $\left(1-\gamma_{i}\right)\left(1-\gamma_{j}\right), \gamma_{i}\left(1-\gamma_{j}\right)^{f},\left(1-\gamma_{i}\right)^{f} \gamma_{j}$, and $\gamma_{i} \gamma_{j}$ for no transmission by any node, successful transmission of node $i$, successful transmission of node $j$, and unsuccessful transmission by both nodes, respectively. Steady state equations can be used to compute the state probabilities $\pi(i, j)$.

Expressions for computation of throughput along with the parameter values are summarized in Table 2. Figure 18 summarizes the analytical and simulated results for SSRC category. A perfect match can be seen in the two results for all packet sizes. It may be noted that the aggregate throughput of the two flows is lesser than the aggregate throughput of SC category. This is because of the higher 


\begin{tabular}{|c|c|c|c|c|c|c|}
\hline Sender & A & RTS(A) & CTS (a) & Data (A) & ACK(a) & DIFS \\
\hline Receiver & a & RTS(A) & CTS (a) & Data (A) & ACK(a) & DIFS \\
\hline Sender & B & & CTS (a) & \multicolumn{3}{|c|}{ NAV } \\
\hline \multirow[t]{2}{*}{ Receiver } & b & RTS (A) & \multicolumn{4}{|c|}{ NAV } \\
\hline & & Channel & sed idle & Channel busy & Signale & on \& SIFS \\
\hline
\end{tabular}

Fig. 17 SSRC channel view

transmission losses and longer binary exponential backoffs, attributed to the higher value of conditional packet loss probability.

\subsection{Asymmetric sender receiver connected (ASRC)}

In this category, the two senders $A$ and $B$ are outside the transmission range of each other. The receiver of flow $B b$ is within the transmission range of sender $A$ while receiver of flow $A a$ is either within carrier sensing range or outside the range of sender $B$. This results in different view of channel for each flow. If RTS frame is transmitted by sender $A$, it is received by receiver $b$, which sets the NAV and remains silent for the entire transmission of the flow $A a$. The transmission of flow $A a$ can be unsuccessful if sender $B$ starts the RTS transmission during the interval between RTS and CTS frames of flow $A a$, which is sensed idle by sender $B$. In case of topology in Fig. $6 \mathrm{~b}$, this interval increases by the duration of CTS frame because sender $B$ cannot sense the activity of receiver $a$. This information is used to compute collision probability of flow $A a$. This behavior of the category is significantly different from AIS category proposed by Garetto et al. [5] where conditional packet loss probability of flow $A a$ is zero because of assumption that the two senders are outside the range of each other. Note that transmission of flow $B b$ in all these scenarios will not be successful given the fact that receiver $b$ has set the NAV after receiving RTS frame from sender $A$. Flow $B b$ can have a successful transmission only when the RTS frame from sender $B$ is initiated while flow $A a$ is

Table 2 Parameters (SSRC computation)

\begin{tabular}{ll}
\hline Parameter & Value \\
\hline Throughput & $\frac{p_{s} T_{s}}{p_{s} T_{s}+p_{c} T_{c}+p_{l} \sigma+p_{s} T_{s}}$ \\
$p_{S}$ & $\frac{\sum_{i, j} \pi_{(i, j} \gamma_{i}\left(1-\gamma_{j}\right)^{f}}{\sum_{i, j} \pi_{(i, j)}\left(\gamma_{i}\left(1-\gamma_{j}\right)^{f}+\gamma_{i} \gamma_{j}\right)}$ \\
$p_{C}$ & $\frac{\sum_{i, j} \pi_{(i, j)} \gamma_{i} \gamma_{j}}{\sum_{i, j} \pi_{(i, j)}\left(\gamma_{i}\left(1-\gamma_{j}\right)^{f}+\gamma_{i} \gamma_{j}\right)}$ \\
$p_{l}$ & $\sum_{i, j} \pi_{(i, j)}\left(1-\gamma_{i}\right)\left(1-\gamma_{j}\right)$ \\
$\gamma_{i}$ & $\frac{2}{W_{i}+1}$ \\
$f$ & ceil((SIFS +6$) / \sigma)=2$ \\
Throughput & $0.0011 \mathrm{pkts} / \mu \mathrm{s}$ \\
\hline
\end{tabular}

in back-off stage. In this case, sender $A$ senses RTS frame and assuming the channel to be busy, it does not initiate transmission. Subsequently, it receives CTS frame and sets the NAV resulting in busy period for flow $A a$ and successful transmission on flow $B b$. The probability of this event is computed by considering the available transmission opportunities for sender $B$ that can lead to successful RTS transmission.

To compute the throughput of the two flows, first of all, the transmission opportunities for flow $B b$ where a successful transmission can occur are considered. A successful transmission on flow $B b$ can only take place if sender $B$ can transmit entire RTS frame during signal extension + DIFS interval and the back-off period of sender $A$. This interval is shown as $D+i \sigma$ in Fig. 19. Note that the SIFS interval between frames is also sensed idle by sender $B$ but is too small for complete RTS transmission and can be ignored. Further note that for topology in Fig. 6b, CTS and ACK packet transmissions are also sensed as idle for sender $B$; however, a transmission during this interval will not lead to successful transmission because of the fact that receiver $b$ can sense the transmissions from receiver $a$. Conditional packet loss probability is the inverse of the probability that sender $B$ successfully transmits RTS frame

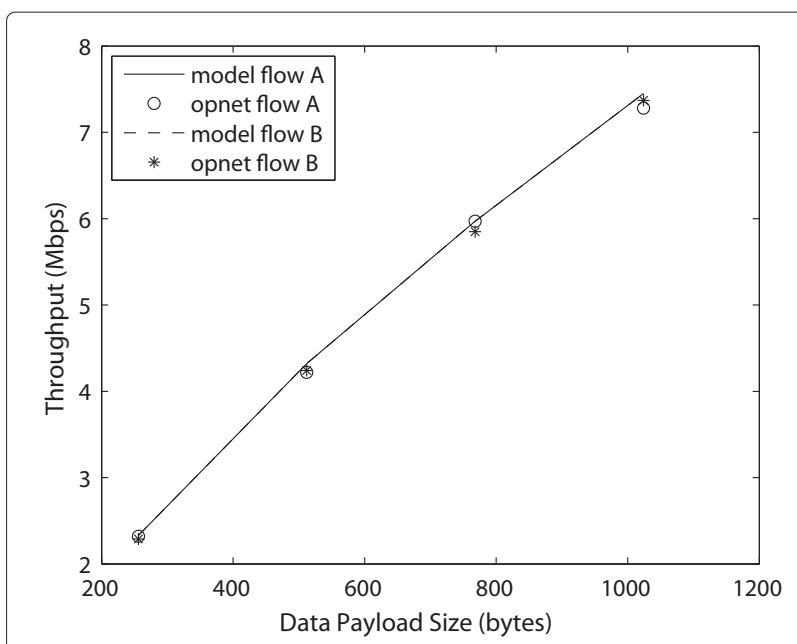

Fig. 18 Per flow throughput of SSRC 


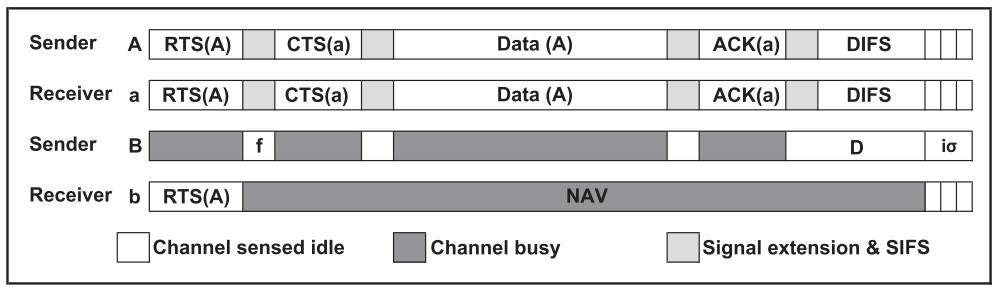

Fig. 19 ASRC channel view

during interval $D+i \sigma$. Garetto et al. have computed this probability using the expression

$$
p_{B}=1-\frac{2\left(\max \left(0, D+\sum_{i=0}^{W_{0}} i \sigma\right)\right)}{W_{0}\left(2 T_{s}+\left(W_{0}-1\right) \sigma\right)}
$$

where $D=$ signal extension + DIFS. Using this equation, packet loss probability of flow $B b$ can be computed in terms of all known variables. Replacing $p_{B}$ in Eq. 9 gives transmission probability $\tau_{B}$ for flow $B b$. Throughput computation of flow $B b$ also requires the value of busy probability $b_{B}$ which is equal to the transmission probability $\tau_{A}$ of flow $A a$. Therefore, we need to compute the transmission probability of flow $A a$ in order to compute the throughput of flow $B b$.

Transmission probability $\tau_{A}$ of flow $A a$ is dependent upon the conditional packet loss probability $p_{A}$. In case of ASRC category, RTS frame transmitted by sender $A$ is sensed by sender $B$ as busy period. However, signal extension + SIFS interval following RTS transmission is sensed as idle by sender $B$. If sender $B$ initiates RTS transmission during this event, it will result in unsuccessful reception of CTS from $a$ at sender $A$, which is the event of collision for flow $A a$. Therefore, probability of packet loss for flow $A a$ can be computed by modeling the probability of the event of RTS transmission by sender $B$ during interval signal extension + SIFS between RTS and CTS transmission by flow $A a$. This event can be modeled as one-dimensional Markov model with $m$ states. The expressions in Table 2 are valid for the purpose with the difference of number of states and the variable $\gamma_{j}$ replaced by $\tau_{B}$. Note that variable $f$ includes additional interval of CTS for the topology in Fig. 6b. Conditional packet loss probability for flow $A a$ is given by the expression for $p_{c}$. Computed value can be used to compute the value of $\tau_{A}$ using Eq. 9. Given that busy probability $b_{B}$ of flow $B b$ is equal to the transmission probability $\tau_{A}$ of flow $A a$ and busy time is equal to $T_{s}$ - DIFS - signal extension, all parameters for throughput computation of flow $B b$ are known. Equation 10 can be used to get the throughput value for flow $B b$.
Busy probability $b_{A}$ of flow $A a$ is given in terms of throughput $\left(T_{B}\right)$ of flow $B b$ as

$$
b_{A}=\frac{\tau T_{s} T_{B}+(1-\tau) \sigma T_{B}}{(1-\tau)\left(1+\sigma T_{B}-T_{b} T_{B}\right)}
$$

The throughput of flow $A a$ can be computed using Eq. 10 in terms of all known parameters. Figure 20 shows the throughput of both flows using analytical and simulated results for different packet sizes. It can be seen that there is a huge imbalance of throughput between two flows for all packet sizes with flow $B b$ severely suffering. This category can be considered as the main cause of bottleneck links in a general multi-hop wireless network.

\subsection{Receiver connected (RC)}

Receiver connected topologies have the interference interactions $A B, A b$, and $a B$ as not connected while interference interaction $a b$ is connected. The MAC behavior of the two topologies belonging to this category is slightly different, although the throughput achieved by the two flows in both categories is the same. In case of topology in Fig. 8a, interference interaction $A B$ is sensing. This means that sender $B$ can sense the channel to be busy during RTS transmission of sender $A$ as shown in Fig. 21.

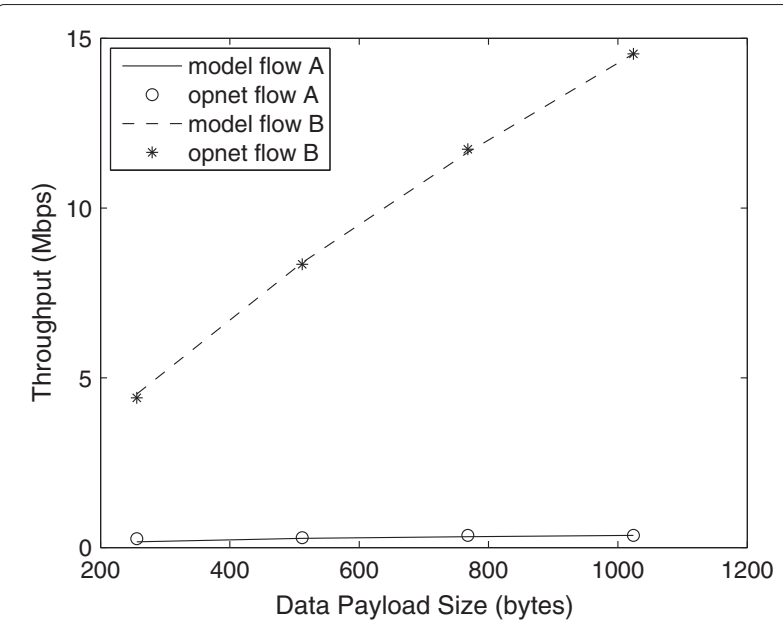

Fig. 20 Per flow throughput of ASRC 


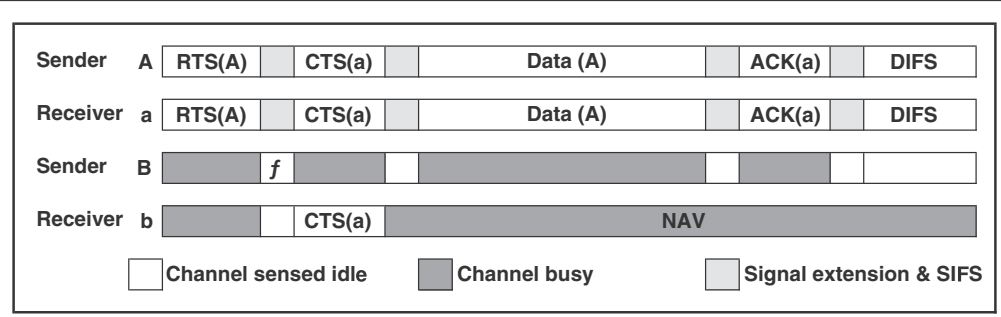

Fig. 21 Channel view of RC (scenario I)

However, signal extension + SIFS interval following RTS transmission is sensed as idle and sender $B$ can initiate its own RTS transmission during this interval, resulting in a collision. Conditional packet loss probability of both flows can be computed by considering this event. Analysis for SSRC category can be used for the purpose with $f=\operatorname{ceil}(($ signal extension $+\operatorname{SIFS}) / \sigma)=2$ and busy period to be equal to $T_{s}$ - DIFS. On the other hand, for topology in Fig. 8b, interference interaction $A B$ is disconnected. Therefore, RTS frame transmitted by sender $A$ is not sensed as busy period by sender $B$ and vice versa. As a result, the interval $f$ for which the sender $B$ must not transmit for transmission of flow $A a$ to be successful is given by $f=\operatorname{ceil}(($ RTS + SIFS $) / \sigma)=8$ as shown in Fig. 22 . This results in higher conditional packet loss probability for both flows. However, the RTS and DATA frames transmitted by sender $A$ are not received by sender $B$ as busy period while the CTS and ACK frames transmitted by receiver $a$ are sensed by sender $B$ as busy. Therefore, event of channel being busy as sensed by sender $B$ is twice the transmission event of sender $A$. The busy interval is equal to CTS $=\mathrm{ACK}$, which is much smaller compared to $T_{s}$ - DIFS. The updated throughput expression becomes

$$
\text { Throughput }=\frac{p_{s} T_{s}}{p_{s} T_{s}+p_{c} T_{c}+p_{I} \sigma+2 p_{s} \mathrm{CTS}}
$$

Figure 23 shows the achievable throughput for the two flows averaged for both topologies. The two flows get almost equal share of throughput. However, the aggregate throughput is lesser than the SC category as well as SSRC category because of higher packet loss probability.

\subsection{Symmetric not connected (SNC)}

Topologies belonging to this category do not have any of the interference interactions as connected. Placement of flow $B b$ within the carrier sensing range of flow $A a$ is possible within a large area of carrier sensing range. Therefore, the distances $d(A, B), d(a, B), d(A, b)$, and $d(a, b)$ can be as small as slightly greater than transmission range and as large as exactly equal to carrier sensing range (which is $\approx 2.7$ times the transmission range) or even outside carrier sensing range. The throughput of the two flows under this category is driven by the fact that frames transmitted by an interferer from closer location within carrier sensing range are sensed as busy periods. On the other hand, the frames transmitted by the interferer at relatively distant location within carrier sensing range may cause errors but otherwise can be rejected as interference, without making the channel busy. Keeping this in view, the MAC behavior can be divided into two parts. The first part comprises of region where a node can sense the frame transmission from other node as busy period. The second part comprises of the region where such transmissions cause errors; however, signal strength is not high enough to make channel busy. Empirical analysis shows that area around a sender up to $r_{\mathrm{csr}}-0.5 r_{\mathrm{tr}}$ comprises of the first part while the presence of interfering nodes within the region beyond this threshold form the second part. Significant area of occurrence of the topology in Fig. 10a exists in the first part. For all remaining topologies belonging to this category, approximately half of the area of occurrence lies within the first part while the remaining half of the occurrence area lies in the second part.

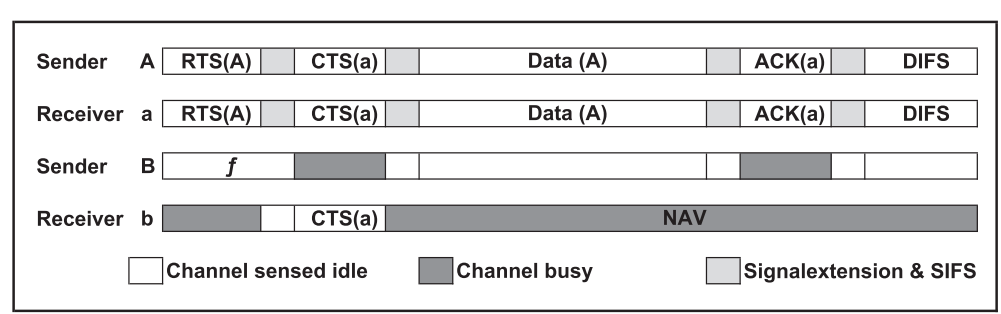

Fig. 22 Channel view of RC (scenario II) 


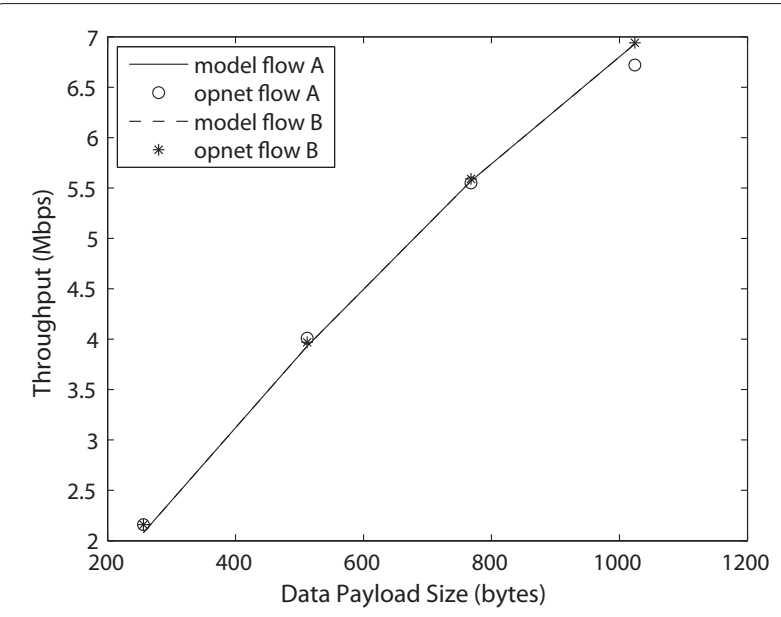

Fig. 23 Per flow throughput of RC

Within near sensing range, the MAC behavior of this category is the same as the MAC behavior of RC category with two differences. First, in case of RC, CTS frame from one receiver is successfully received by the other receiver, which sets the NAV for rest of the transmission. On the other hand, in case of SNC category, CTS is not successfully received, resulting in busy period at other receiver only for the duration of CTS. However, the idle interval of signal extension + SIFS between the four frames (RTS, CTS, DATA, and ACK) is not big enough to allow any successful transmission. Therefore, the entire transmission on one flow results in errors on receiver of alternate flow in case any frame is transmitted by alternate flow. Effectively, this difference in behavior does not affect the throughput. Second, the interval $f$ during which the flow $B b$ must not have a transmission in order to have a successful transmission on flow $A a$ varies for different topologies of this category. Similarly, the busy interval and collision interval vary for each topology. The parameters for different topologies are summarized in Table 3. and as such the analysis of RC category remains valid for the first region of SNC category. Once again, the difference in MAC behavior within category does not impact the throughput of the flows. Figure 24 shows analytical and simulated throughput. The two flows achieve nearly equal throughput while a perfect match can be observed between simulated and analytical results.

The impact of interference from far sensing range on throughput of the flows can be estimated by considering the received signal strength, its impact on bit error rate and packet error rate. Ideal channel conditions are assumed where the only factor affecting the unsuccessful reception of frame is the interference from the other flow. Although extremely simplifying, even under this assumption, throughput of the two flows can be predicted
Table 3 Parameters (SNC computation)

\begin{tabular}{lll}
\hline Topology & Parameter & Value \\
\hline a,b & $f$ & ceil((signal extension + SIFS $) / \sigma)$ \\
a,b & $T_{b}$ & $T_{s}-$ DIFS \\
a,b & $T_{c}$ & RTS + SIFS + CTS + DIFS \\
$c, d$ & $f$ & ceil((RTS + SIFS $) / \sigma)$ \\
$c, d$ & $T_{b}$ & CTS/ACK \\
$c, d$ & $T_{c}$ & RTS + DIFS \\
e,f,g & $f$ & ceil((CTS + SIFS - signal extension $) / \sigma)$ \\
$e, f, g$ & $T_{b}$ & (DATA + ACK $-2^{*}$ signal extension) 0.5 \\
e,f,g & $T_{c}$ & RTS + SIFS + CTS + DIFS - signal extension
\end{tabular}

accurately. This assumption allows the computation of packet error probability for RTS frame. Near sensing range analysis is used as basis while the conditional packet loss probability and busy probability of the flows are adjusted by the packet error probability to achieve the throughput of the two flows that interfere from within far sensing range.

Friis transmission equation [22] is used to compute the received signal strength from the intended transmitter and the interfering transmitter at the receiver. Ratio of signal received from intended sender and the interfering transmitter gives the signal-to-noise ratio (SNR). SNR can be used to get bit error rate using well-known BER curves and eventually packet error probability using the size of RTS frame. The packet error probability decreases exponentially from distance $r_{\mathrm{csr}}-0.5 r_{\mathrm{tr}}$ to $r_{\mathrm{csr}}$ with the values at two boundaries to be 0.97 and 0.03 , respectively. Throughput of the flows for 512 bytes of packet size as a function of distance $d(A, B)$ is shown in Fig. 25. Empirical values have also been plotted at selective points. It can be seen that

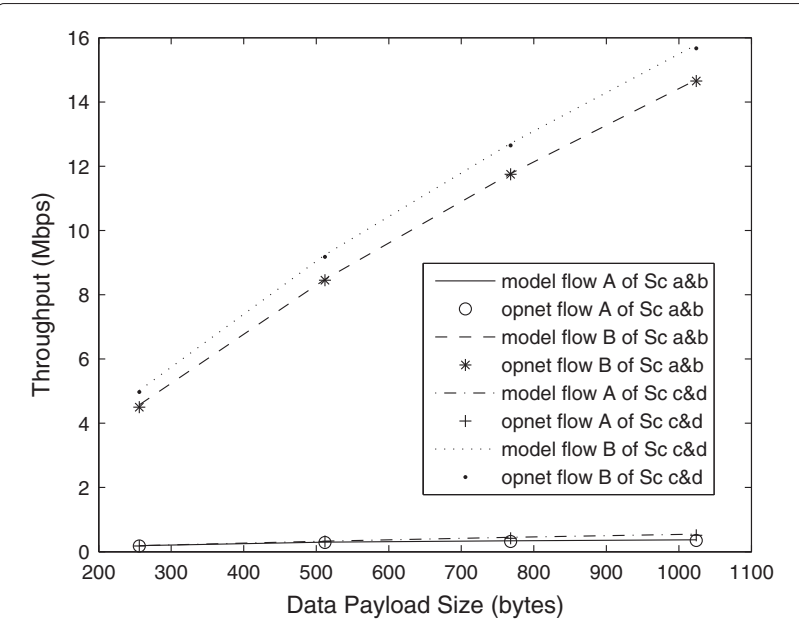

Fig. 24 Per flow throughput of SNC (near) 


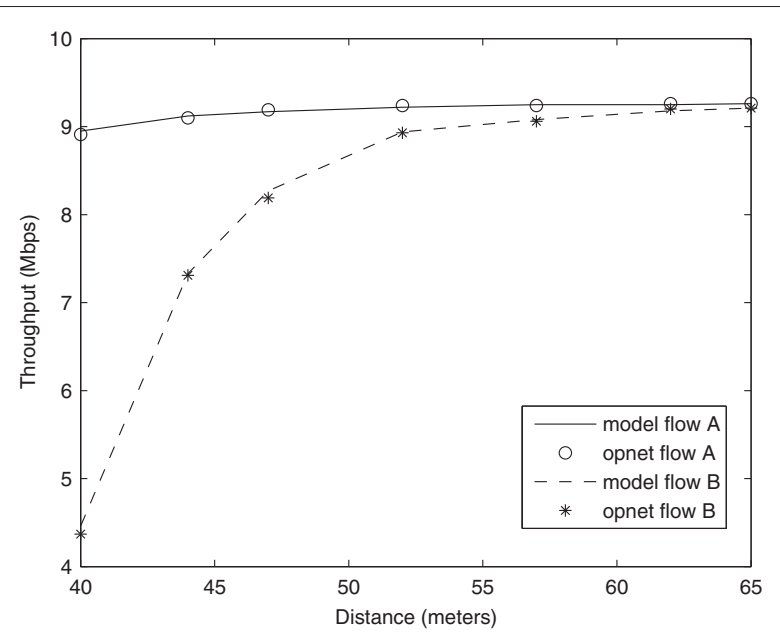

Fig. 25 Per flow throughput of SNC (far)

the throughput exponentially increase within the plotted range, starting from minimum value equivalent to near sensing range and ending at near independent throughput for two flows.

\subsection{Asymmetric not connected (ANC)}

Topologies belonging to this category have interference interaction $A b$ as sensing while the interference interaction $a B$ is disconnected. Like ASRC category, asymmetric channel view of the two flows results in imbalance among achievable throughput with one flow $A a$ getting dominant portion of channel capacity while the other flow $B b$ getting negligible throughput. Similar to SNC category, the distance between nodes of two flows affects the achievable throughput. The MAC behavior of the category predicts the initial throughput of the two flows at minimum possible distance. With the increasing distance, the impact of one flow on the other is mitigated, gradually making two flows independent of each other. For the throughput computation, the MAC behavior is defined first and the throughput of the two flows is computed. Subsequently, the results because of the SNR-based adjustments to the behavior are reported, similar to SNC category.
For the analysis purposes, interference interaction $A b$ is considered as sensing while the interference interaction $a B$ is considered to be disconnected. The conditional packet loss probability of flow $B b$ can be computed by considering the interval during which RTS transmission from $B$ will be received successfully by $b$. This interval is given by $D+i \sigma$ where $D=$ signal extension + DIFS and $i$ is the average number of back-off slots. There is a difference between MAC behavior of the topologies. For topologies in Fig. 13a, b, sender $A$ can sense RTS transmission from sender $B$; therefore, $B$ only needs to initiate the RTS transmission within the specified interval for its transmission to be successful. On the other hand, for topologies in Fig. 13c, d, Senders $A$ and $B$ are outside the sensing range; therefore, sender $B$ must complete the transmission of the entire RTS frame during the specified interval for the transmission to be successful. For the later case, $D$ is updated to $D=$ signal extension + DIFS - RTS. Given the value of the interval, Eq. 11 can be used to compute the conditional packet loss probability for flow $B b$. The busy probability of two group of topologies also differs. For topologies in Fig. 13a, b, busy probability $b_{B}=\tau_{A}$ because of the fact that $B$ can sense the transmissions of $A$. Busy interval is equal to $T_{s}-$ (SIFS + ACK + DIFS). On the other hand, busy probability of flow $B b$ for topologies in Fig. 13c, d is zero. Throughput of flow $B b$ can be computed in terms of all known parameters using Eq. 10, provided the value of $\tau_{A}$ is known.

To compute the throughput of flow $A a$, the probabilities $p_{A}, b_{A}$ and the interval $T_{b}$ are required. For topologies in Fig. 13a, b, a collision on flow $A a$ occurs when sender $B$ starts a transmission following the RTS frame transmission by sender $A$. In this case, the channel is sensed idle by sender $B$ for the interval 2signal extension + 2SIFS + CTS because $B$ is outside carrier sensing range of $a$. A transmission attempt by $B$ results in collision at $A$ if RTS is transmitted by $B$ within interval signal extension + SIFS + CTS. Markov model used to compute conditional packet loss probability for ASRC category can be used to compute $p_{A}$ with $f=\operatorname{ceil}(($ signal extension + SIFS + CTS $) / \sigma)$ as shown in Fig. 26. Busy probability $b_{A}$ is computed as a function of

\begin{tabular}{|c|c|c|c|c|c|c|c|}
\hline Sender & A & RTS(A) & CTS(a) & Data (A) & ACK(a) & DIFS & \\
\hline Receiver & a & RTS(A) & CTS(a) & Data (A) & ACK(a) & DIFS & \\
\hline Sender & B & & $f$ & & & D & io \\
\hline \multirow[t]{2}{*}{ Receiver } & b & & & & & & \\
\hline & & Channel & sed idle & hel busy & \multicolumn{3}{|c|}{ Signalextension \& SIFS } \\
\hline
\end{tabular}

Fig. 26 ANC channel view (scenario II) 
throughput of flow $B b$ using Eq. 12. Busy interval for these topologies is given by $T_{s}$ - DIFS. Equation 10 can be used to compute the throughput of flow $A a$ using all known parameters.

In case of topologies in Fig. 13c, d, conditional packet loss probability is zero, resulting in $\tau_{A}=2 /(W+1)$. Successful transmissions on flow $B b$ result in busy intervals for sender $A$ when receiver $b$ transmits CTS/ACK frames. Therefore, busy probability $b_{A}=2 \tau_{B}$ and busy interval are equal to CTS/ACK. With all known parameters, throughput can be computed using Eq. 10. Figure 27 shows the analytical and simulated results for throughput of the two flows for two different types of topologies. It can be noted that although the MAC behavior is slightly different, there is not much difference in achieved throughput for two types. The imbalance between the throughput of the two flows is also obvious from the results.

With the throughput values at near sensing range available, the distance and SNR-based analysis similar to SNC category is applied for computation of throughput of the two flows with increasing distance. Figure 28 shows the achievable throughput as a function of distance between sender $A$ and receiver $b$ for packet size of 512 bytes. It can again be observed that although the analysis technique is based on simplifying assumption, the predicted throughput closely matches the simulated results.

\section{Conclusions}

This work has investigated the MAC behavior of two single hop IEEE 802.11 standard-based interfering flows. All possible two-flow topologies have been identified using realistic transmission and carrier sensing ranges. The identified categories have been divided into six categories based on the MAC behavior as well as geographic placement of the four interfering nodes. Closed-form

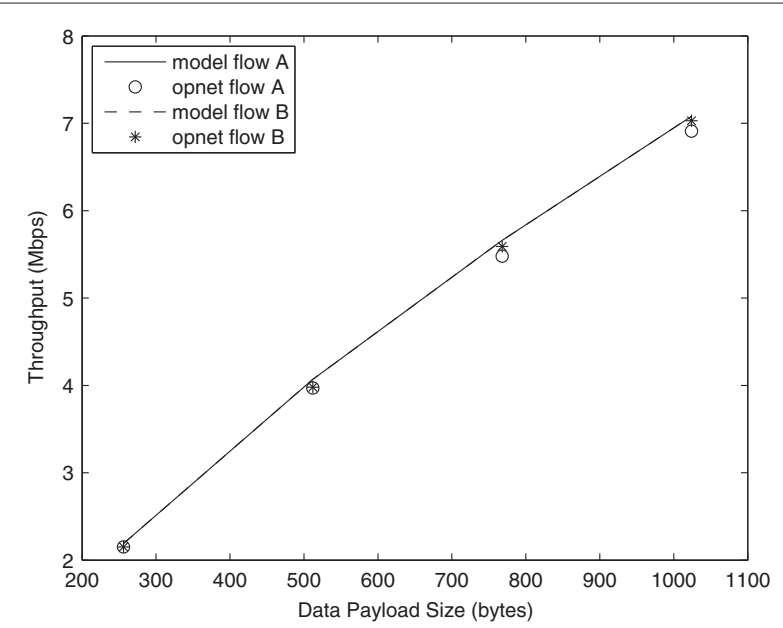

Fig. 27 ANC per flow throughput

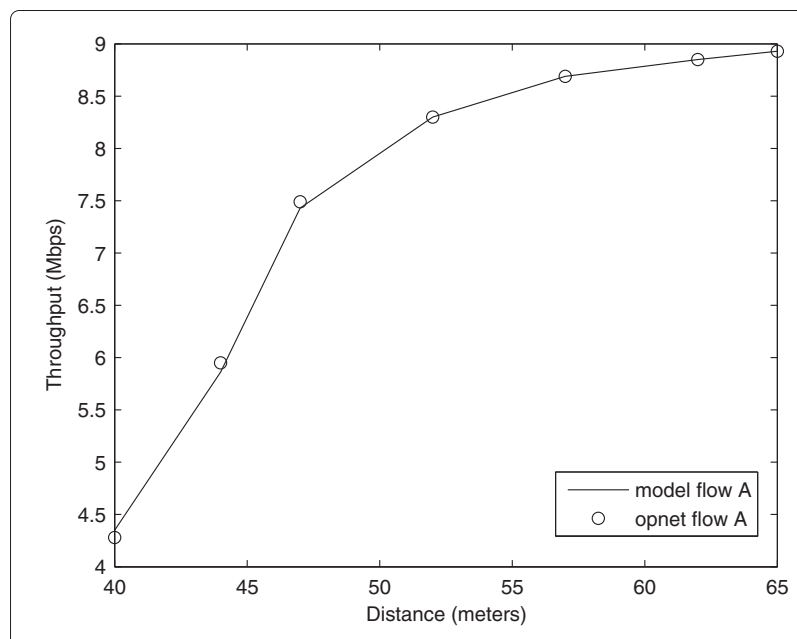

Fig. 28 Per flow throughput for ANC with increasing distance $d(A, b)$

expressions for occurrence probabilities of all identified categories have been computed to show that all categories have significant probability of occurrence in a general multi-hop wireless network. MAC behavior of each category is thoroughly discussed with the key observation that the presence of interfering nodes within the carrier sensing range has significant impact on the behavior and the throughput of five out of six identified categories. Based on the MAC behavior, extensive throughput computations are performed for both flows under each category. This work completes the research efforts towards defining the MAC behavior of two-flow topologies and its impact on the throughput of links. The work can be extended to general capacity analysis of multi-hop wireless networks and can serve as the basis for modified MAC protocol that can better mitigate the impact of interference, specifically the interference from within carrier sensing range.

\section{Competing interests}

The authors declare that we have competing interests with the following; Dr Saquib Razak, Assistant Teaching Professor, Computer Science, email address: srazak@qatar.cmu.edu.

Received: 26 July 2015 Accepted: 21 January 2016

Published online: 09 February 2016

References

1. P Gupta, PR Kumar, The capacity of wireless networks. Inform. Theory IEEE Trans. 46(2), 388-404 (2000)

2. J Tang, G Xue, W Zhang, in Proceedings of the 6th ACM International Symposium on Mobile Ad Hoc Networking and Computing. Interference-aware topology control and gos routing in multi-channel wireless mesh networks (ACM, 2005), pp. 68-77

3. L Qiu, Y Zhang, F Wang, MK Han, R Mahajan, in Proceedings of the 13th Annual ACM International Conference on Mobile Computing and Networking. A general model of wireless interference (ACM, 2007), pp. 171-182

4. K Medepalli, FA Tobagi, in Proceedings IEEE INFOCOM 2006. 25TH IEEE International Conference on Computer Communications. Towards performance modeling of IEEE 802.11 based wireless networks: a unified framework and its applications, (2006) 
5. M Garetto, J Shi, EW Knightly, in Proceedings of the 11th Annual International Conference on Mobile Computing and Networking. Modeling media access in embedded two-flow topologies of multi-hop wireless networks (ACM, 2005), pp. 200-214

6. M Garetto, T Salonidis, EW Knightly, Modeling per-flow throughput and capturing starvation in CSMA multi-hop wireless networks. IEEE/ACM Trans. Netw. (TON). 16(4), 864-877 (2008)

7. S Razak, V Kolar, NB Abu-Ghazaleh, Modeling and analysis of two-flow interactions in wireless networks. Ad Hoc Netw. 8(6), 564-581 (2010)

8. S Razak, NB Abu-Ghazaleh, in Ad-hoc, Mobile and Wireless Networks. Self-interference in multi-hop wireless chains: geometric analysis and performance study (Springer, Berlin Heidelberg, 2008), pp. 58-71

9. RR Boorstyn, A Kershenbaum, B Maglaris, V Sahin, Throughput analysis in multihop CSMA packet radio networks. Commun. IEEE Trans. 35(3), 267-274 (1987)

10. G Bianchi, Performance analysis of the IEEE 802.11 distributed coordination function. Selected Areas Commun. IEEE J. 18(3), 535-547 (2000)

11. F Tobagi, L Kleinrock, Packet switching in radio channels. Part II-the hidden terminal problem in carrier sense multiple-access and the busy-tone solution. Commun. IEEE Trans. 23(12), 1417-1433 (1975)

12. G Alfano, M Garetto, E Leonardi, in INFOCOM, 2011 Proceedings IEEE. New insights into the stochastic geometry analysis of dense CSMA networks (IEEE, 2011), pp. 2642-2650

13. $M L i, H L i u, H$ Tan, $M$ Yang, Performance and interference analysis of 802.11 g wireless network. Int. J. Wirel. Mob. Netw. (IJWMN), 165-177 (2013)

14. M Dinitz, in INFOCOM, 2010 Proceedings IEEE. Distributed algorithms for approximating wireless network capacity (IEEE, 2010), pp. 1-9

15. S Kawade, TG Hodgkinson, V Abhayawardhana, in Vehicular Technology Conference, 2007. VTC-2007 Fall. 2007 IEEE 66th. Interference analysis of $802.11 \mathrm{~b}$ and $802.11 \mathrm{~g}$ wireless systems (IEEE, 2007), pp. 787-791

16. S Kawade, TG Hodgkinson, in Vehicular Technology Conference, 2008. VTC Spring 2008. IEEE. Analysis of interference effects between co-existent $802.11 \mathrm{~b}$ and $802.11 \mathrm{~g}$ wi-fi systems (IEEE, 2008), pp. 1881-1885

17. S Weber, JG Andrews, N Jindal, An overview of the transmission capacity of wireless networks. Commun. IEEE Trans. 58(12), 3593-3604 (2010)

18. L Fu, SC Liew, J Huang, Effective carrier sensing in CSMA networks under cumulative interference. Mobile Comput. IEEE Trans. 12(4), 748-760 (2013)

19. S Vitturi, L Seno, F Tramarin, M Bertocco, On the rate adaptation techniques of ieee 802.11 networks for industrial applications. Ind. Inform. IEEE Trans. 9(1), 198-208 (2013)

20. D Qiao, S Choi, KG Shin, Interference analysis and transmit power control in IEEE 802.11 a/h wireless lans. IEEE/ACM Trans. Netw. (TON). 15(5), 1007-1020 (2007)

21. A Kumar, E Altman, D Miorandi, M Goyal, in INFOCOM 2005. 24th Annual Joint Conference of the IEEE Computer and Communications Societies. Proceedings IEEE. New insights from a fixed point analysis of single cell IEEE 802.11 wlans, vol. 3 (IEEE, 2005), pp. 1550-1561

22. Wikipedia contributors, "Friis transmission equation," Wikipedia, The Free Encyclopedia, https://en.wikipedia.org/w/index.php?title= Friis_transmission_equation\&oldid=672456199 (Accessed 29 January 2016)

\section{Submit your manuscript to a SpringerOpen ${ }^{\circ}$ journal and benefit from:}

- Convenient online submission

- Rigorous peer review

- Immediate publication on acceptance

- Open access: articles freely available online

- High visibility within the field

- Retaining the copyright to your article

Submit your next manuscript at $\gg$ springeropen.com 